\title{
A VIRTUAL LAB TO VISUALIZE THE PERFORMANCE OF THE HOPFIELD'S NEURAL NETWORK FOR ASSOCIATIVE CONTENT- ADDRESSABLE MEMORY
}

\author{
J. Montenegro Joo \\ Virtual Dynamics / Virtual Labs: Science \& Engineering \\ Calle 14 -572, Las Magnolias, Surco, Lima 33, Perú,
}

\begin{abstract}
A software to appreciate the performance of the Hopfield's Neural Network (Associative Content-Addressable Memory) has been developed and applied to computer synthesized images. A rather small network has been created and applied to four sets of training-remembrances.

The software allows the user to become familiar with associative memory computer simulations, it also provides knowledge on the work of a neural net, hence this computer program may be used as a training tool (teach learn) on neural networks. This software makes evident that a straightforward application of neural networks is in the field of pattern recognition and image reconstruction; it also serves as an introduction to more advanced and complex neural nets. This report is aimed at understanding the performance and potentials of a neural network, it may also foster the interest of students in cybernetics.

The Hopfield neural network is important to physicists because it is closely related to the Ising Spin Glass model of magnetism, the learned memories in the net stand for the low energy states in the Ising model. A set of images (shown in this report) is included in the software, however it also accepts those made by the user.
\end{abstract}

Keywords: Cybernetics, Artificial Intelligence, Artificial Neural Networks, Hopfield, Pattern Recognition, Image Reconstruction, Ising, Magnetism.

\section{Resumen}

Con la finalidad de apreciar la ejecución de la red de neuronas de Hopfiled (memoria asociativa), se ha desarrollado un software y se ha aplicado a imágenes sintéticas. Una pequeña red de neuronas ha sido creada y aplicada a cuatro conjuntos de entrenamiento-remembranzas.

El software permite al usuario familiarizarse con la simulación en computadoras de la memoria asociativa, también proporciona conocimientos sobre la operación de una red de neuronas, de modo que el software puede ser usado como una herramienta para enseñar-aprender redes de neuronas. El software hace evidente que una aplicación directa de las redes de neuronas, es el reconocimiento de patrones y la reconstrucción de imágenes, también sirve como una introducción a redes mas avanzadas y complejas. Este reporte apunta a entender la ejecución y potencialidades de una red de neuronas, puede también estimular el interés de los estudiantes en la Cibernética.

La red de Hopfield es importante para los físicos, pues está muy relacionada al modelo de Vidrios de Spin de Ising del magnetismo, las memorias que aprende la red, equivalen a los estados de mínima energía en el modelo de Ising. El programa incluye un conjunto de imágenes (mostrado en este reporte), sin embargo, también acepta aquellas suministradas por el usuario.

Palabras clave: Cibernética, Inteligencia Artificial, Redes de Neuronas artificiales, Hopfield, Reconocimiento de patrones, Reconstrucción de imágenes, Ising, Magnetismo. 


\section{Introduction}

\subsection{Associative Memory}

It is due to associative memory that when a physicist and many times a non-physicist sees " $\mathrm{E}=\mathrm{mc}^{3}$ ", he/she recalls that it actually is $\mathrm{E}=$ $\mathrm{mc}^{2}$, remembers the name Einstein and imagines an old man with disordered long white hair, others may also remember that famous photograph where he is showing out his tongue.

\subsection{What is a Neural Network?}

The fruit fly is to genetics, like the Bohr's atom is to modern physics, like the Hopfield's network is to the field of neural networks (NN). A NN ${ }^{1-6}$ is a set of interconnected units or neurons, see Fig.1, there are synapses (connections) with some particular strength $\mathrm{W}$ between neurons. The state of neuron $\mathrm{k}$ is $\mathrm{S}_{\mathrm{k}}$ and $\mathrm{Wmn}$ is the strength of the synapse connecting neurons $\mathrm{m}$ and $n$. The allowed values of the states $S_{i}$ may be discrete, like $-1,+1$ or $-1,0,+1$, etc, or continuous, like $S_{\mathrm{i}}$ in the interval 0 to 1 or -1 to +1 . Generally the output of a neuron depends on the total input $h_{i}$ (a weighted sum) it receives:

$$
h_{i}=\sum_{j} \omega_{i j} S_{j}
$$

the synapses $\mathrm{W}_{\mathrm{ij}}$ may be excitatory, when $\mathrm{W}_{\mathrm{ij}}>0$, (Neurons $\mathrm{i}$ and $\mathrm{j}$ are in Parallel states), or inhibitory, when $\mathrm{W}_{\mathrm{ij}}<0$, (Neurons $\mathrm{i}$ and $\mathrm{j}$ are in Antiparallel states), and the allowed values of $\mathrm{W}_{\mathrm{ij}}$ may be discrete or continuous and bounded or unbounded. The values of the synapses $\mathrm{W}_{\mathrm{ij}}$ control the performance of a $\mathrm{NN}$, these have initially any random value and then they are updated as the network learns some piece of information, so that their updated values represent the learned information, this means that the information grasped by a $\mathrm{NN}$ is stored in the weights (synapses) connecting units (neurons).

Neural Networks have been applied mainly to pattern recognition ${ }^{8-9,14-15}$ (image and sound), in the industry, defence systems, speech recognition, optical character recognition, identification of finger prints, etc

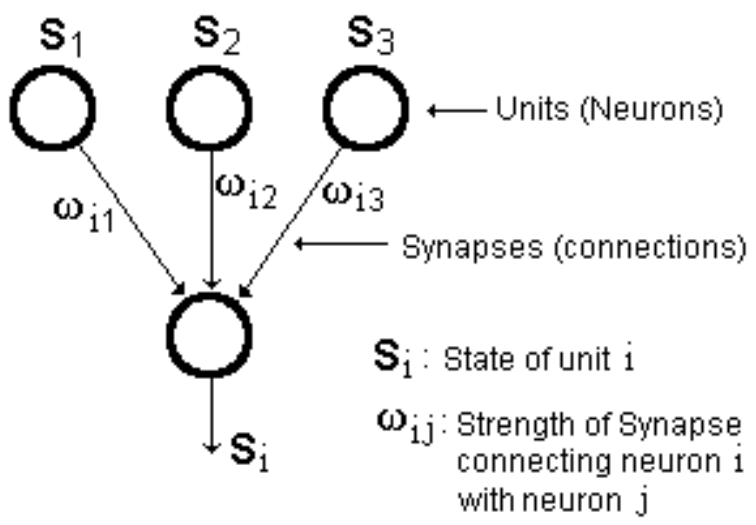

Total input to unit $\mathrm{i}: \mathrm{h}_{\mathrm{i}}=\sum_{\mathrm{j}} \omega_{\mathrm{ij}} \mathbf{S}_{\mathrm{j}}$

\section{Fig. 1 A very simple Neural Network}

\subsection{Association from Distorted and Noisy Data}

The recall of stored information by the brain relies rather on associations with previously stored data, than on the order in which the memory was acquired; with conventionally programmed computers this task requires precise knowledge of the memory address. Up to a certain degree, neural networks are able to emulate the performance of the brain. Despite the simplicity of the work of the NN reported in this paper, it can not be achieved by a conventionally programmed computer, these are not able to operate on distorted, vague and noisy data, at least not in the short time and with the straightforwardness of the NN.

\subsection{The connection between Neural Networks and Magnetism}

Physicists became interested in the field of NN, because the Hopfield model of NN results being a variation of the Ising Spin Glass model of magnetism, where the spins updating protocol is very similar to that used in the Hopfield model, the bonds $\mathrm{J}$ between spins are symmetrical $\mathrm{J}_{\mathrm{mn}}=\mathrm{J}_{\mathrm{nm}}$ and the spins flip one at a time. The Hopfield model includes an Energy function, which cannot increase and whose stable states are associated with the stored patterns. The problem of $\mathrm{NN}$ as a memory devise is the inverse spin-glass problem ${ }^{6}$. Physics themes ${ }^{1-4}$ like Hamiltonians, statistical mechanics, finite temperature dynamics, spins in equilibrium, 
mean field theory, Replica-symmetry breaking, Basins of attraction, etc, have a parallel in the field of $\mathrm{NN}^{10}$ and are very useful to understand them.

\subsection{Types of NN and learning protocols}

There are several types ${ }^{3}$ of NN, like Perceptrons (simple and multiplayer), Adalines, Hopfield networks, Boltzmann machine, Kohonen nets, Neocognitrons, etc. Updating of neurons while the networks learns some piece of information may be synchronous (all $S_{i}$ are changed at once) and asynchronous (only some $S_{i}$ are updated every time, generally on a random basis). Concerning the learning protocols, these may be supervised and unsupervised, and within these there are quite a few algorithms. Researchers have developed plethora ${ }^{7-9}$ of $\mathrm{NN}$ and learning algorithms, depending on their applications, some investigators have also used genetic algorithms to develop the architecture of their networks.

\subsection{Associative Content - Addressable Memory}

In Associative Content-Addressable memory ${ }^{11}$ when an input pattern is presented to the network for recognition, this evolves towards the most similar stored memory (pattern), because this most-similar pattern acts as an Attractor for the network dynamics. The purpose of the autoassociative memory ${ }^{1}$ is the embedding of prespecified patterns or memories into a network for later retrieval. As can be appreciated with the software being reported in this paper, retrieval of information is possible even with partial and/or noisy information.

\subsection{The three steps of a recognition process} are

(1) Training: The NN learns some memories (patterns, images) and stores them into its synapses.

(2) Remembrance: The NN "sees" part of a learned pattern (memory), this partial "view" may even be distorted and noisy.

(3) Recognition: The NN associates "seen" information either Asynchronously (Randomly updating a neuron at a time) or Synchronously (Updating all neurons simultaneously at once), to reconstruct the corresponding memory (pattern).

\section{The Hopfield Neural Network}

Here a description of the Hopfield network ${ }^{11-13}$ is presented.

\subsection{General Features}

- There is only a single layer of neurons (other models have more layers).

- There are feedback connections from each neuron to every other neuron.

- There are no connections from a neuron to itself.

- The weights (strengths) on the connections (synapses) are symmetrical.

- There is an Energy function E.

- The gain of the neurons is controlled by a Sign function.

- The Energy landscape has many local minima, being this the main drawback in terms of engineering applications.

\subsection{Learning in the Hopfield Network}

There are $\mathrm{N}$ interconnected binary neurons, then there are $\mathrm{N} \times \mathrm{N}$ synapses $\mathrm{W}_{\mathrm{ij}}$, of the which a number $\mathrm{N}(\mathrm{N}-1) / 2$ are pairs of symmetric connections $\mathrm{W}_{\mathrm{ij}}=\mathrm{W}_{\mathrm{ji}}$, Every Neuron $\mathrm{Si}$ may be either On or Off, this is: $\mathrm{Si}=+1$ or $\mathrm{Si}=-1$. Assume the number of patterns (memories, images) to be stored is $\mathrm{p}$ and are given by $\xi_{i}{ }^{\mu}= \pm 1 \quad \mu=1,2,3, \ldots p$. Each pattern has $\mathrm{N}$ bits $( \pm 1)$, one for each neuron $i$. The usual way to change the links $\mathrm{W}_{\mathrm{ij}}$ so as to learn the input patterns is the Hebb learning rule, which in the case of pattern $u$ is given by:

$$
\omega_{i j}=\frac{1}{N} \sum_{u=1}^{p} \xi_{i}^{u} \xi_{j}^{u} \quad i, j=1,2, \ldots, N
$$

where $\mathrm{u}=1,2, \ldots, \mathrm{p}$ and $\xi_{i}{ }_{i}$ is the bit $\mathrm{i}$ of pattern $\mathrm{u}$ to be stored, $\omega_{i j}$ is the synapse between neuron $i$ and $j$. The Hebb prescription as it is also known- is a one-shot learning rule, which means that learning takes place in a single operation, with more sophisticated learning 
algorithms a number of iterations-updates are necessary for the information to be grabbed by the network. The new state $S_{i}$ of neuron $i$, this is the network dynamics, is given by

$$
S_{i}^{u}(t+1)=\Theta\left[\sum_{j=1}^{N} \omega_{i j} S_{j}^{u}(t)-T\right]
$$

where $\mathrm{T}$ is a threshold (usually $\mathrm{T}=0$ ), and $\Theta(x)$ is the Sign function

$$
\Theta(x)=\left\{\begin{array}{lll}
+1 & \text { when } & x \geq 0 \\
-1 & \text { when } & x<0
\end{array}\right.
$$

The Energy function is

$$
E=-\frac{1}{2} \sum_{i j} \omega_{i j} S_{i} S_{j}
$$

in Spin Glasses this is the Magnetic Interaction Energy and involves all the spins in the system, in neural networks this is an abstract quantity associated to the information content of the net. The Energy surface has local minima at the points $S_{i}=\xi_{i}^{\mu}$. The Energy is used during learning when there are iterations involved, in every iteration $\mathrm{E}$ goes down as the $\mathrm{NN}$ learns.

\subsection{Storage Capacity.-}

In the current case patterns are $15 \times 15$ pixels, which means that the number of neurons (one for each pixel) is $\mathrm{N}=15 \times 15=225$. According to Hopfield, if there are small errors in the stimuli, the maximum number of patterns $p_{\max }$ that can be stored in a network with $\mathrm{N}$ neurons, is $\mathrm{p}_{\max }=$ $0.15 \mathrm{~N}, \quad\left(\mathrm{p}_{\max }=33.75\right.$, for $\left.\mathrm{N}=225\right)$, he reached to this conclusion by means of computer simulations. Then Amit et al ${ }^{16}$ through the theoretical Replica Method showed that $\mathrm{p}_{\max }=$ $0.138 \mathrm{~N},\left(\mathrm{p}_{\max }=31.05\right.$, when $\left.\mathrm{N}=225\right)$. Mc Eliced et al ${ }^{17}$ concluded that for $\mathrm{p}<\mathrm{N} /(4 \ln \mathrm{N})$, the Hopfield $\mathrm{NN}$ is able to reconstruct all learned patterns with no error. In the current case:

$$
p=\frac{N}{4 \operatorname{Ln} N}=10.38
$$

which means that the number of patterns must be at most 10, but this is as well as the patterns are fully orthogonal, in real life patterns are far from being sharing this feature and this number is greatly reduced, for this reason the network of this work operates on at most 4 patterns. For orthogonal patterns $\xi_{i}^{\mu}$ and $\xi_{i}^{v}$, their scalar (dot) product satisfies:

$$
\sum_{i=1}^{N} \xi_{i}^{\mu} \xi_{i}^{v}=0 \quad \text { for } \quad \mu \neq v
$$

\section{The Experiments}

In every experiment the network is first trained with a set of image inputs, these are learned as memories, then a number of remembrances (stimuli) is presented one at a time to the net and this, by means of association, recalls the more similar image memorized during training. After developing the computer program, four independent experiments were executed.

\subsection{Experiment 1}

Fig. 2 shows the set of (four) memories (input set) the network is trained with (square, rhombus, triangle, arrow). As it can be seen in figs A1 through A10, the 10 presented stimuli (remembrances) are not so similar to the images in the training set, they are distorted, sometimes vague and include noise, however, the network after being stimulated reconstructs the most similar memory (Recognition).

Note that even though the stimuli are confusing, noisy and distorted images, the neural network reconstructs the original objects. 

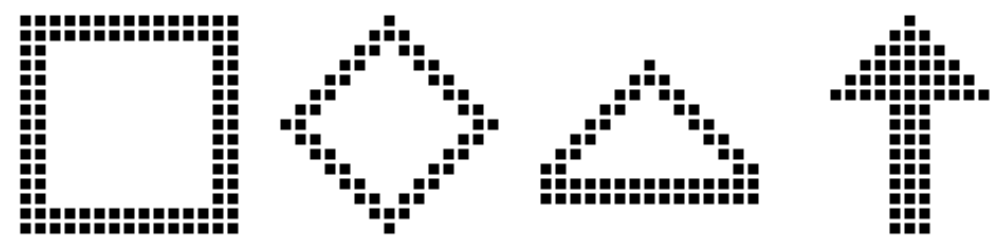

Fig 2. Training set A: 4 objects
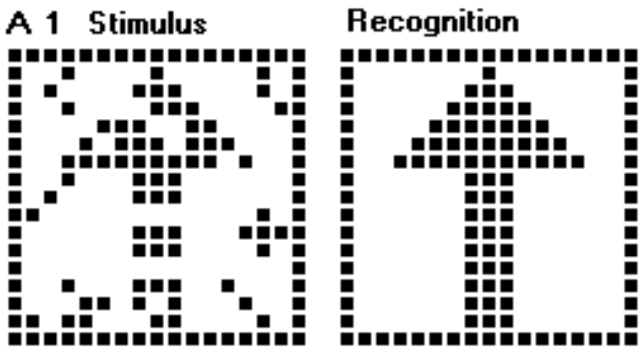

A 3 Stimulus
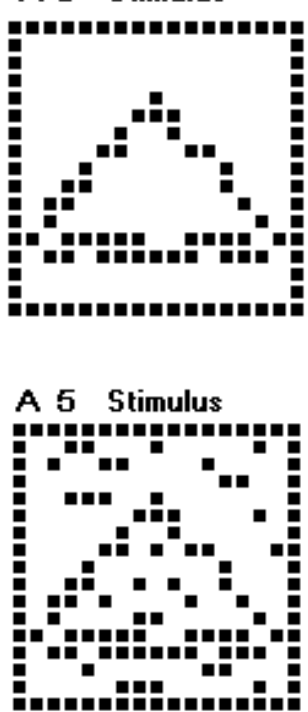

Recognition

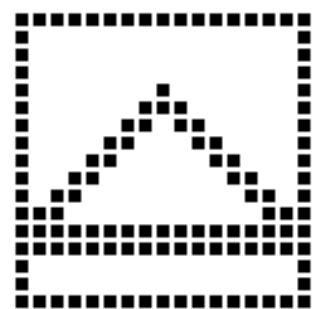

Recognition
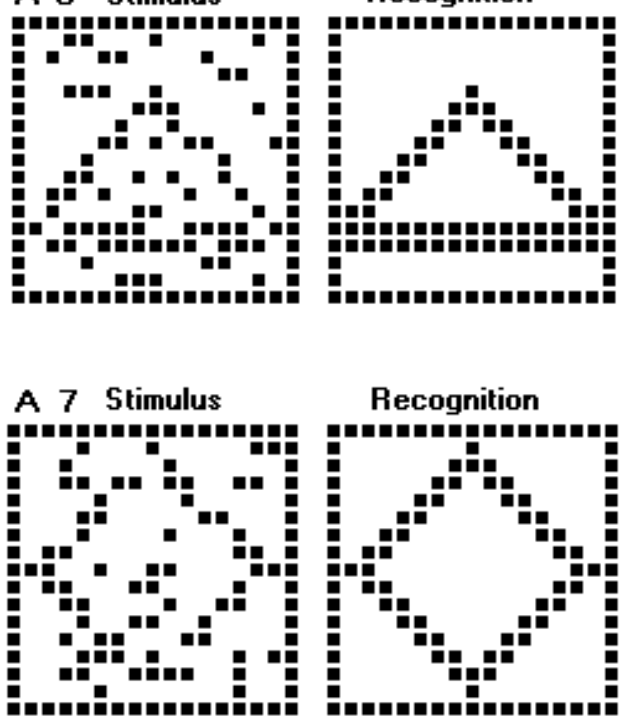

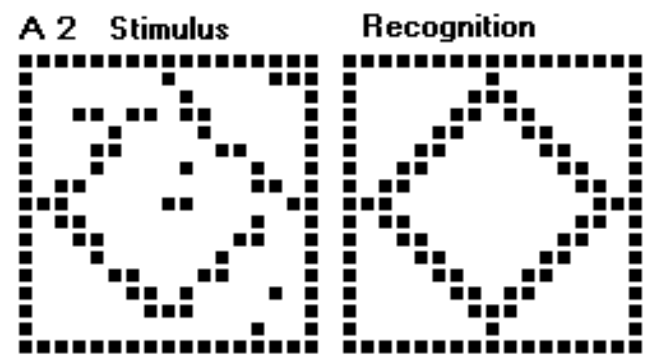

A 4 Stimulus

Recognition
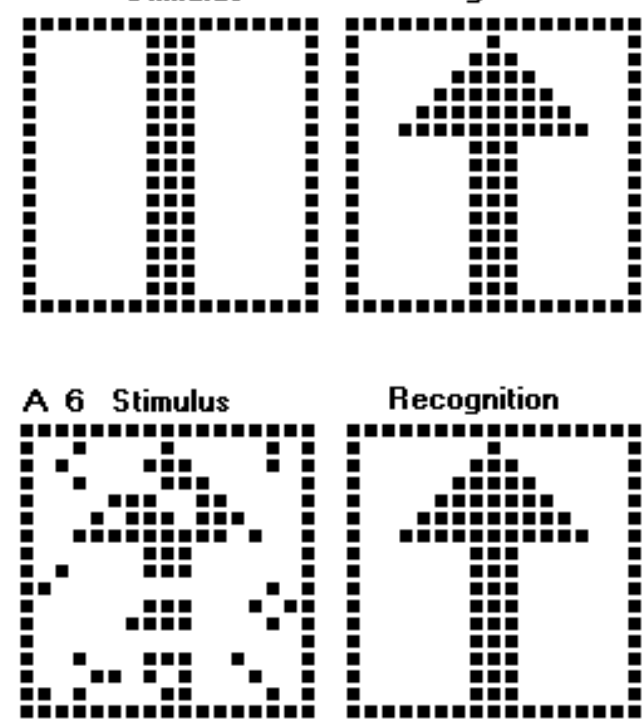

Recognition
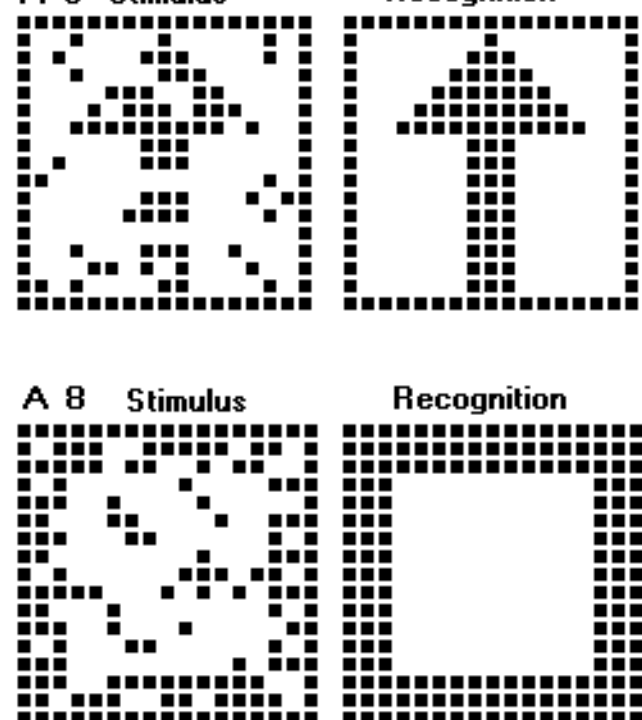

Recognition

PIIIII

但

身

9日e

P 


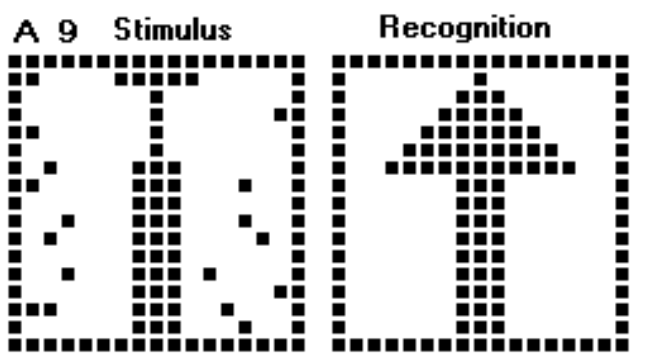

\subsection{Experiment 2}

The Network was trained with two images (Fig. 3), a happy face and a plus sign, then it was motivated with the stimuli in images B1 through $\mathrm{B} 10$, the reconstructed images appear as Recognition. It can be seen that notwithstanding the remembrances (stimuli) are in many case vague, distorted and noisy, the network is able to make the right association. In all cases the stimuli are sad faces or part of them, however the network has reconstructed the original happy faces of the input. Stimulus B2 as well as B7 show part of a horizontal line, but not in the position of that of the cross, however the network recognises the cross in its correct

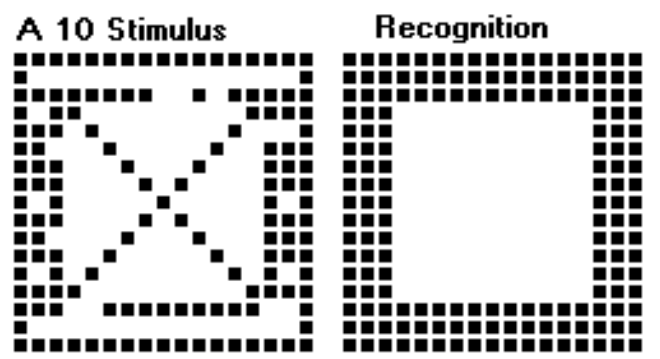

(original) position. In Fig. B4, the system discards the swastika (which is a cross) and reconstructs the original cross. In Fig. B8 the stimulus are two diagonal lines and the net seems to "think" that any two lines must appear crossing themselves. In computer graphics a straight line is not only a succession of points but also a succession of line-segments. Notice in fig. B10, that the stimulus is not the plus sign of the corresponding training set, but rather the closed line around it, apart of noise, there are only 8 scattered dots belonging to the original plus sign, however the network associates it to the correct memory.
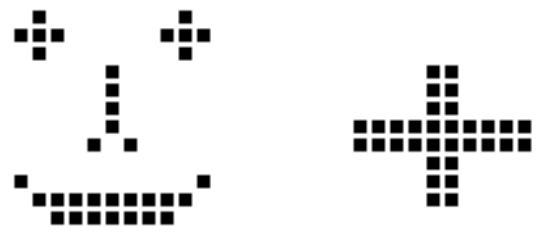

Fig. 3 Training set $B \quad 2$ objects

B 1 Stimulus

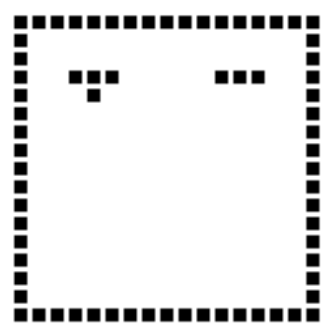

Recognition

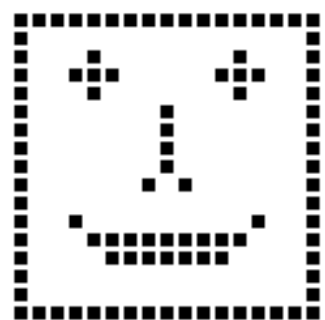

B 2 Stimulus

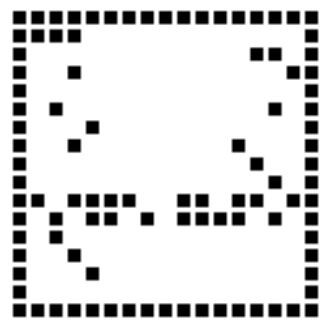

Recognition

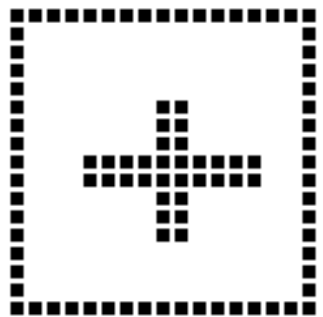




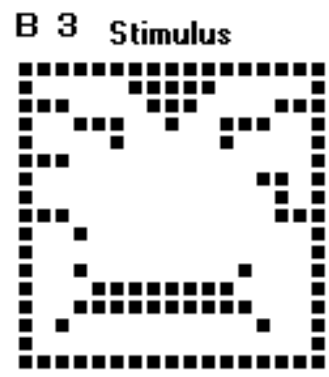

Recognition

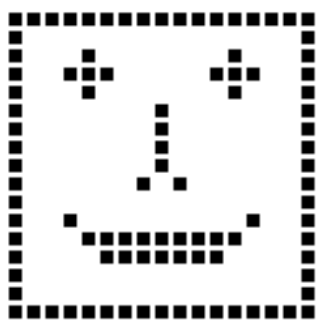

B 5 Stimulus

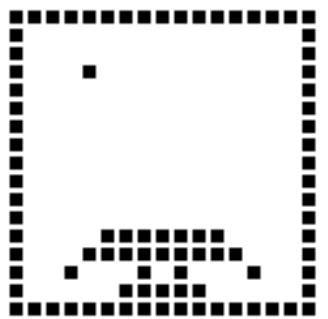

Recognition

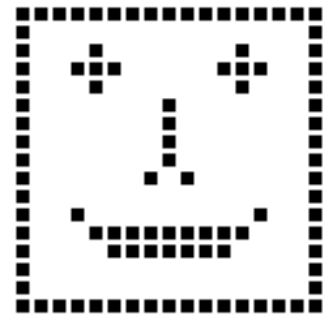

B 7

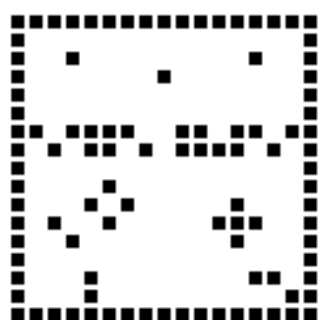

Recognition

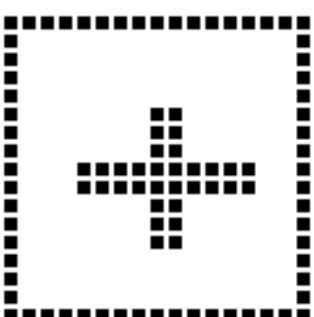

B 9 Stimulus

Recognition

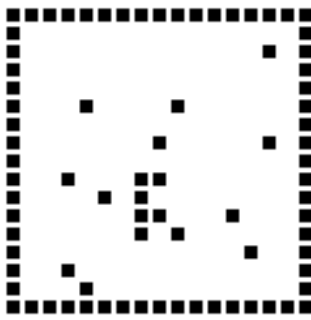

B 4 Stimulus

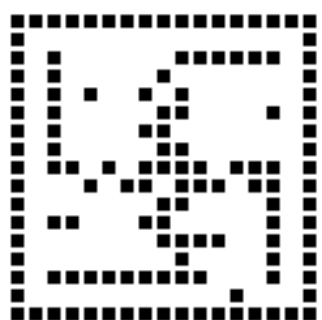

B 6 Stimulus

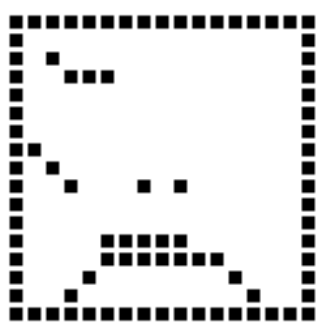

В 8

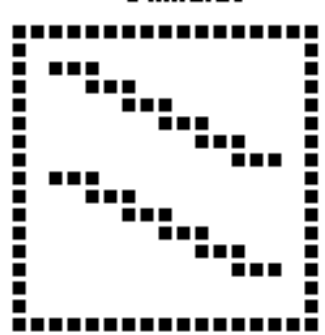

B 10 Stimulus

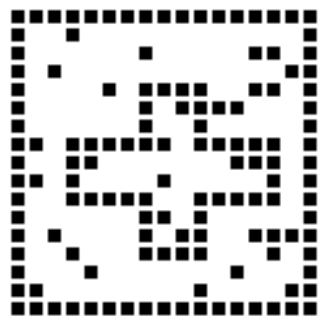

Recognition

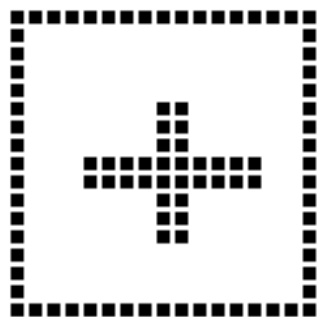

Recognition

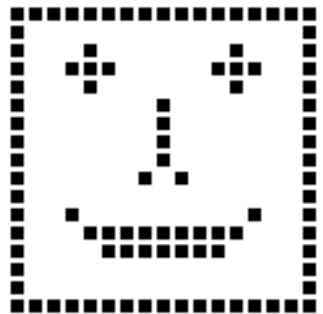

Recognition

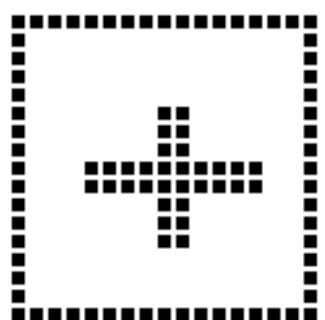

Recognition

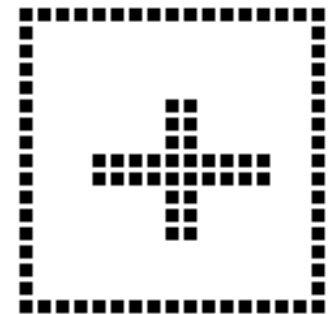

\subsection{Experiment 3}

Fig. 4 shows the input set for experiment 3, the input set (3 images) now is that used in experiment 2 (Fig 3) but includes an additional memory, the square. In this case, the 10 stimuli (remembrances) were those from B1 to B10, all were successfully reconstructed (recognized), except those shown in C7, C8 and C10. Notice

that reconstructions $\mathrm{C} 7$ and $\mathrm{C} 8$ are both a combination of the happy face and plus sign, and that reconstruction $\mathrm{C} 10$ includes parts of the three training memories. 

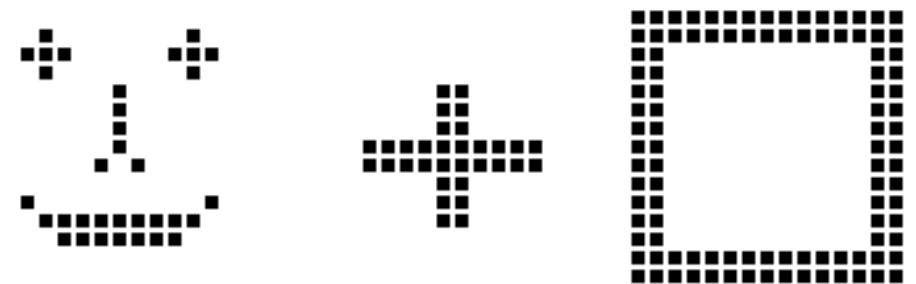

Fig 4 Training set $c: 3$ objects
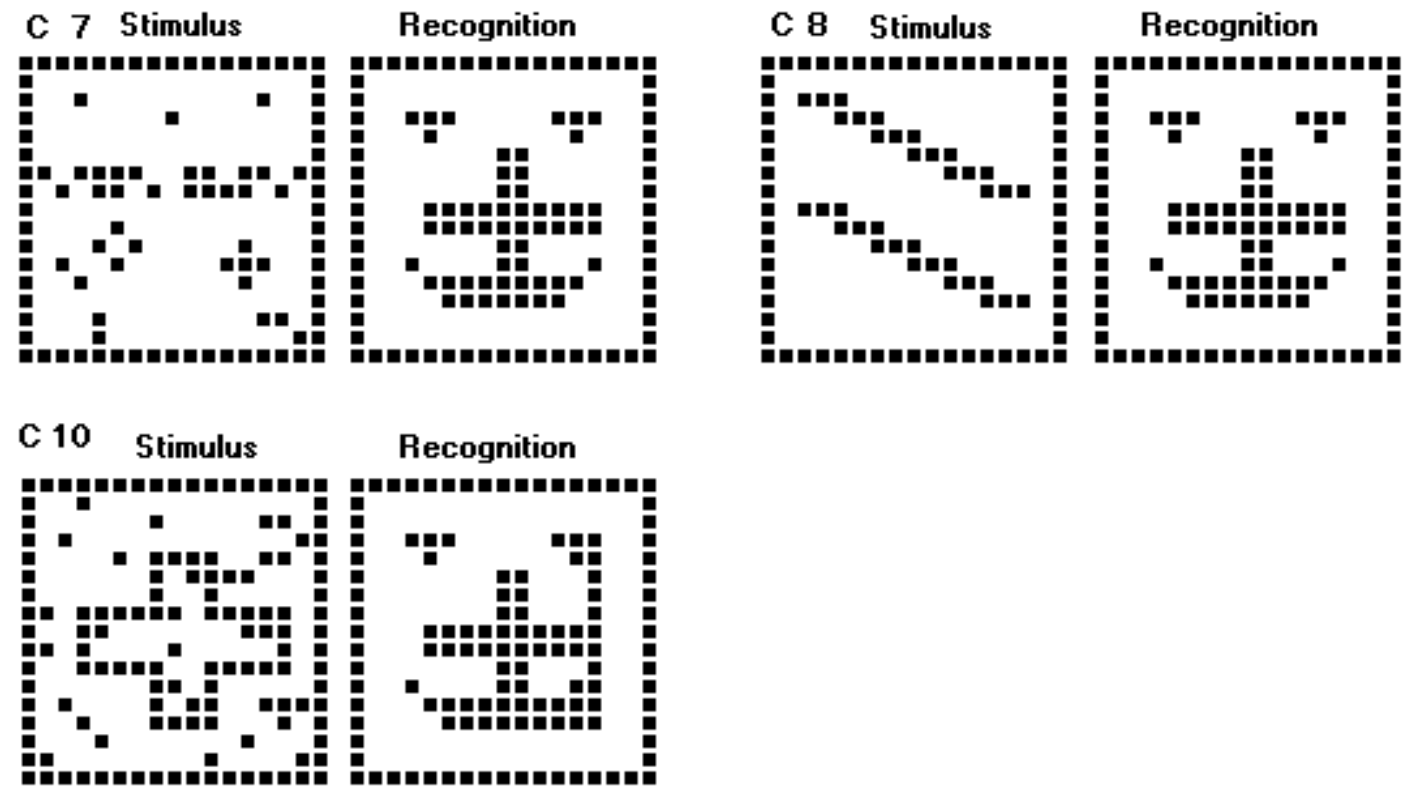

Recognition

\subsection{Experiment 4}

recognized, the other eight were not. As examples of the ill-recognized memories,

The input set of four images is shown in Fig. 5, the 10 remembrances used as stimuli are those recognitions D8 and D10 are shown.

images shown in B1 through B10. In this case only two inputs (B3 and B4) were successfully

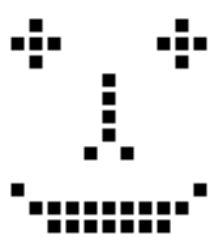

Fig 5

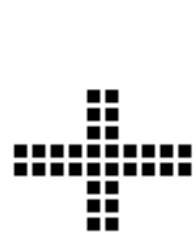

Training set D:
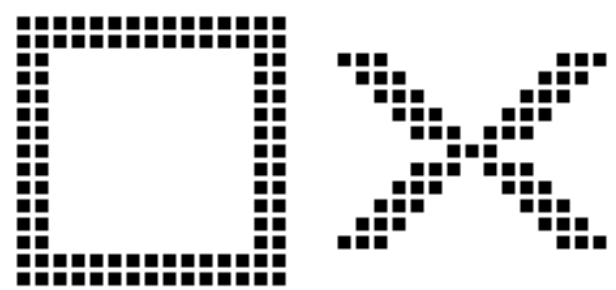
4 objects 


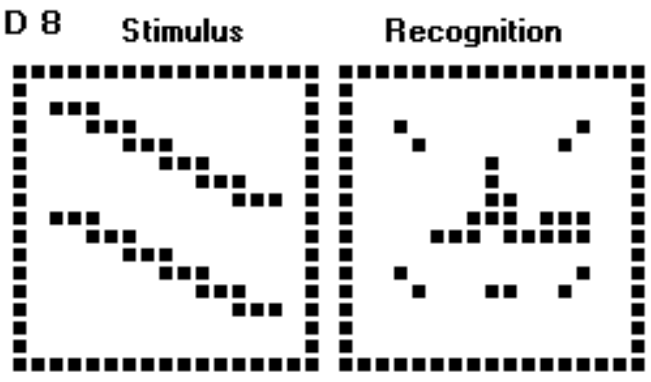

\section{Conclusions}

The mission was successfully accomplished: a software to use as a demonstrator of the Hopfield model for associative memory was developed and it operates effectively. This software may be used to learn about associative memory in general, to understand what a neural network is and to see that a network like that of Hopfield can be used in pattern recognition and image reconstruction, even with distorted, noisy and vague images.

Physicists familiar with the Spin Glass Ising model of magnetism may see that the Hopfield network is just a variation of that. Based on this NN software development experience, larger softwares to operate on more powerful computers may be developed. In the figures shown the recognition has been Synchronous (ordered neuron updates), the Asynchronous case (random updates) is not shown, however the results are the same but take longer because being it random, many neurons may be activated more than once and this takes computer time.

The experiments have shown that when the network tries to learn input patterns that are somehow related among them (mathematically speaking, not orthogonal), the recognition presents problems, the system becomes confused and memories too close to each other tend to merge ${ }^{11}$, a common phenomenon among humans and maybe also among animals.

\section{ACKNOWLEDGEMENT}

The development of this Neural Network software would not have been possible without the help of Miss Marlene Gonzalez Reyes, who

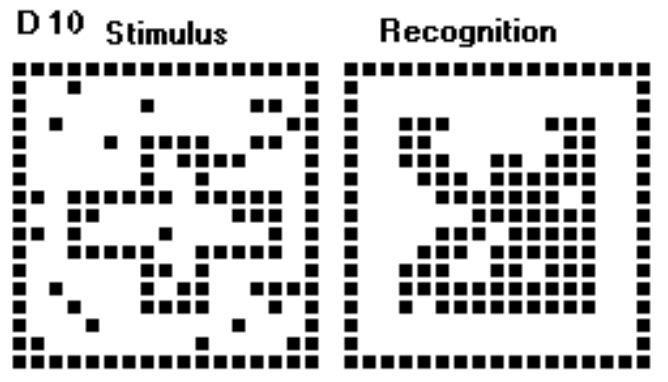

created the computer synthesized objects and helped with the computer programming.

\section{References}

[1] Neural Nets, Richard Palmer, Pre-print, 1989.

[2] Neural Computation, Lecture Notes. J.A. Hertz, R.G. Palmer, Duke University, 1988.

[3] Neural Networks, an introduction. B. Muller-J. Reinhardt, Springer-Verlag, 1991

[4] Intro. to the theory of neural computation, Hertz-Krogh, Palmer, Addison Wesley, 1991.

[5] Modelling brain function, the world of attractor neural networks, Daniel Amit, Cambridge Univ. Press, 1989.

[6] Eytan Domany, Neural Networks: A biased overview, Journal of Stat. Phys. Vol 51 Nos 5/6, 1998.

[7] D.R. Hush-B.G. Horne, Progress in supervised neural networks, IEEE Signal Processing Magazine, Jan 1993.

[8] Richard P. Lippmann, Pattern classification using neural networks.

[9] M.W. Roth, Survey of neural network technology for automatic target recognition, IEEE trans on neural nets, Vol 1, No 1, March 1990.

[10] Complex Systems: Fractals, Spin Glasses and Neural Networks, Proceedings of the Internat. Conf. on Complex Systems, ICTP Trieste Italy, July 1991, North-Holland Ed. 
[11] J.J. Hopfield, Neural networks and physical systems with emergent collective computational abilities, Proc. Natl. Acad. Sci. USA, Vol 79, pp 2554-2558, 1982.

[12] J.J. Hopfield, Neurons with graded response have collective computational properties like tose of two-state neurons, Proc. Natl. Acad, Sci., USA, Vol 81 May 1984.

[13] R.J. Mc. Eliece et al, The Capacity of the Hopfield Associative Memory, IEEE transactions on Information Theory, Vol IT33, No 4, July 1987.

[14] Yoh-Han Pao, Neural Net Computing for pattern recognition, Handbook of pattern recognition and computer vision, World Scientific Publishing Co., 1993.
[15] J. Montenegro Joo-L. Da Fontoura Costa, Geometric-transformation-invariant pattern recognition with Hough transforms and Distance-Discriminator Neural Networks. Workshop sobre Computacao de alto desempenho para processamento de sinais, SP, Brasil, 1993.

[16] D.J. Amit - H. Gutfreund - H. Sompolinsky, Statistical Mechanics of Neural Networks near saturation, Annals of physics, No 173, 33-67,1985.

[17] R.J. McEliced - E.C. Posner - E.R. Roderick - S.S. Venkatesh, the capacity of the Hopfield associative memory, IEEE Trans. on Info. Theory, IT-33, 461-482, 1987. 